\title{
Using Event Detection Latency to Evaluate the Coverage of a Wireless Sensor Network
}

\author{
You-Chiun Wang, Kai-Yang Cheng, and Yu-Chee Tseng ${ }^{1}$ \\ Department of Computer Science \\ National Chiao-Tung University \\ Hsin-Chu, 30010, Taiwan, ROC
}

Email:\{wangyc, kycheng, yctseng\}@csie.nctu.edu.tw

\begin{abstract}
A wireless sensor network (WSN) consists of many tiny and low-power devices deployed in a sensing field. One of the major tasks of a WSN is to monitor the surrounding environment and to detect events occurring in the sensing field. Given an event appearing in a WSN, the event detection latency is to model the time that it takes for the WSN to be aware of the event. In this work, we analyze the latency using a probabilistic approach under an any-sensor-detection and a $k$-sensor-detection models, where $k>1$ is an integer. Such an analysis can be used as an index to evaluate a WSN's coverage and thus can help guide the deployment of a WSN. We also develop simulations to verify our analytical results.
\end{abstract}

Key words: ad hoc network, network coverage, pervasive computing, ubiquitous computing, wireless sensor network.

\section{Introduction and Problem Statement}

Wireless sensor networks (WSN) have been intensively studied recently [1]. A WSN consists of many tiny and lower-power sensor nodes, each of which can collect surrounding environmental data and communicate with neighboring nodes. Communications in a WSN typically takes place in an ad hoc manner [2]. Applications of WSNs include surveillance and agriculture, habitat, traffic, and civil infrastructure monitoring [3-7].

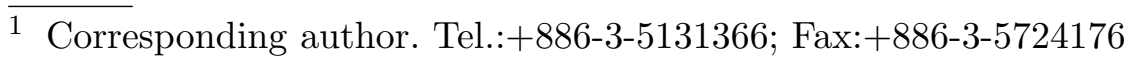




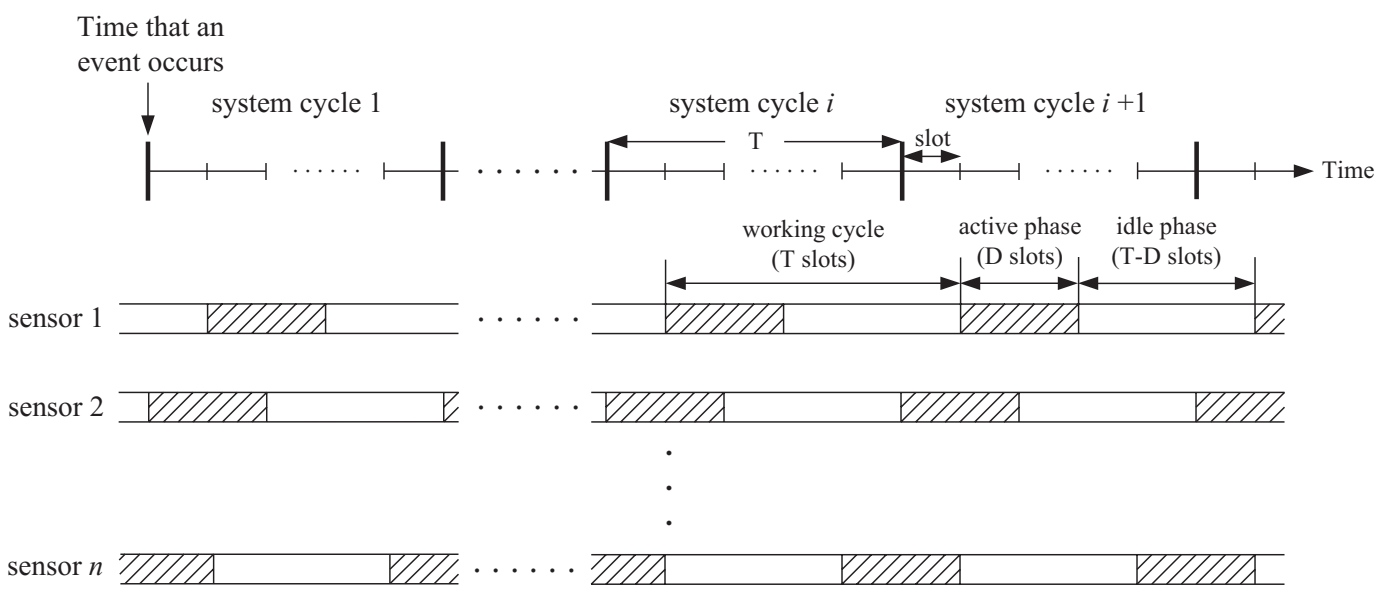

Fig. 1. Modeling of sensors' working cycles.

One of the major tasks of a WSN is to detect events occurring in the sensing field. Given an event appearing in a WSN, the event detection latency is to model the time that it takes for the WSN to be aware of the event. Such latency is an important metric to measure the monitoring capability of a WSN's deployment for real-time applications such as surveillance [8-10] or object tracking [11-13].

We propose our model to analyze the event detection latency. Specifically, we are given a sensing field, on which there are $n$ homogeneous sensors. Each sensor has a sensing distance of $r$. Without loss of generality, we assume that these $n$ sensors form a connected network. To simplify the analysis, we assume that the time axis is divided into fixed-length slots and the working schedule of each sensor is modeled by a sequence of working cycles, each of length $T$ slots. Each working cycle is led by an active phase followed by an idle phase. The former consists of the first $D$ slots, and the latter the rest of the $T-D$ slots. Sensors only conduct detection jobs in their active phases, and go to sleep in idle phases. However, sensors do not synchronize their clocks, so their working cycles are not necessarily aligned. Fig. 1 shows an example. Note that this model can be applied to most of the MAC/network protocols that are proposed for WSN recently. For example, for energy conservation, the Zigbee/IEEE 802.15.4 standard [14] allows a sensor node to wake up and sleep very similarly to our working cycles in Fig. 1. In fact, several other protocols (such as Bluetooth [15] and S-MAC [16]) also have such an awakesleep behavior.

Our objective is to evaluate the detection latency when an event occurs in the sensing field. Note that we make no assumption on the locations of events. To take errors into account, we also assume that in an active slot, a sensor has a probability of $p$ to successfully detect the occurrence of an event if the event is within this sensor's sensing range. To simplify the analysis, we assume that $p$ is a constant but not a function of the distance between a sensor and the 
event $[17,18]$. We consider two detection models in this work:

- any-sensor-detection model: To capture the event, the network needs at least one sensor to successfully detect the event.

- $k$-sensor-detection model: To capture the event, the network needs at least $k$ sensors to successfully detect the event, where $k>1$. (The value of $k$ is application-dependent. For example, positioning protocols using triangulation [19-21] require at least three sensors.)

\section{Analysis of Event Detection Latency}

To facilitate the calculation of the event detection latency, we establish a system clock, which starts at the instant when the event appears. The system time is also slotted and each set of continuous $T$ slots forms a system cycle, as shown in Fig. 1. Suppose that an event appears at location $(x, y)$ in the sensing field. Let $M(x, y)$ be the number of sensors whose sensing ranges cover location $(x, y)$. Consider the time slots that these $M(x, y)$ sensors start their new working cycles in a system cycle. We can classify them into $T$ groups such that the $i$ th group contains the $m_{i}$ sensors that start their working cycles at the $i$ th slot in a system cycle, $i=1 . . T$. For example, in Fig. 1, sensor 1 belongs to group 2, sensor 2 belongs to group 1, and sensor $n$ belongs to group $T$. Clearly, $\sum_{i=1}^{T} m_{i}=M(x, y)$. Taking all combinations of $m_{i}$ 's into consideration, the event detection latency under this particular $M(x, y)$ can be written as

$$
\begin{aligned}
& \operatorname{Latency}(M(x, y))=\sum_{m_{1}=0}^{M(x, y)} \sum_{m_{2}=0}^{M(x, y)-m_{1}} \cdots \\
& \sum_{m_{T-1}=0}^{M(x, y)-\left(m_{1}+\cdots+m_{T-2}\right)}\left(\frac{M(x, y) !}{m_{1} ! \cdots m_{T} !} \times\left(\frac{1}{T}\right)^{M(x, y)}\right) \times \delta\left(m_{1}, \cdots, m_{T}\right),
\end{aligned}
$$

where the first term is the probability to observe a particular combination $\left(m_{1}, \ldots, m_{T}\right)$, and the second term $\delta\left(m_{1}, \cdots, m_{T}\right)$ is the expected latency for this particular combination.

As the event may appear in any location $(x, y)$ inside the sensing field, we have to consider all possible $M(x, y)$. Thus, the overall expected latency of the WSN can be expressed as

$$
\begin{aligned}
E_{T, D} & =\int_{x} \int_{y} \operatorname{Latency}(M(x, y)) d x d y \\
& =\sum_{i=0}^{n} \operatorname{Prob}[M(x, y)=i] \cdot \operatorname{Latency}(i) .
\end{aligned}
$$


When the event appears in a $i$-covered region, it will be sensed by $i$ sensors (i.e., $M(x, y)=i)$. Therefore, $\operatorname{Prob}[M(x, y)=i]$ is the ratio of areas that are $i$-covered in the sensing field. So Eq. (1) can be simplified as

$$
E_{T, D}=\sum_{i=0}^{n} \frac{A_{i}}{A} \cdot \operatorname{Latency}(i),
$$

where $A$ is the area of the sensing field, and $A_{i}$ is the total area in $A$ in which each point is covered by exactly $i$ sensors.

In Sections 2.1 and 2.2, we will show how to compute $\delta\left(m_{1}, \cdots, m_{T}\right)$ under our two detection models, respectively. Table 1 summarizes the notations used in this work.

\subsection{Any-Sensor-Detection Model}

Under this model, the event is considered to be captured by the network if any sensor successfully detects its existence. Let $x_{i}$ be the number of active sensors at the $i$ th slot, $i=1 . . T$. These $x_{i}$ sensors are composed of three types of sensors: (1) sensors which turn into active at the $i$ th slot, (2) sensors which turn into active between the first and the $(i-1)$ th slots, and (3) sensors which turns into active before the first slot. Note that case (2) can be true if $D>1$ and $i>1$, while case (3) can only occur when $i<D$. This leads to

$$
x_{i}=m_{i}+\sum_{j=1}^{\min (D-1, i-1)} m_{i-j}+\sum_{j=0}^{D-i-1} m_{T-j} .
$$

We also define $x_{a T+b}$ as the number of active sensors at the $(a T+b)$ th slot for any $a \geq 1$. Since cycles repeat every $T$ slots, we have $x_{a T+b}=x_{b}$.

The probability that there is at least one sensor successfully detecting the event in the first slot is $\left(1-(1-p)^{x_{1}}\right)$. For $i \geq 2$, the probability that the event is not detected in the first $(i-1)$ slots but is successfully detected in the $i$ th slot is $\left(1-(1-p)^{x_{i}}\right)(1-p)^{x_{1}+\ldots+x_{i-1}}$. Hence, as the time goes to infinity, the expected detection latency under the any-sensor-detection model is

$$
\begin{aligned}
\delta\left(m_{1}, \cdots, m_{T}\right)= & \sum_{a=0}^{\infty} \sum_{b=1}^{T}(a T+b) . \\
& \left(1-(1-p)^{x_{b}}\right)(1-p)^{a \times\left(x_{1}+\cdots+x_{T}\right)+x_{1}+\cdots+x_{b-1}} .
\end{aligned}
$$

Eq. (2) contains an infinite number of expressions. The following theorem shows that it will converge. 
Table 1

Summary of notations used in this work.

\begin{tabular}{|c|c|}
\hline Notations & Definition \\
\hline$n$ & number of sensors in the sensing field \\
\hline$k$ & $\begin{array}{l}\text { minimum number of sensors required to successfully detect } \\
\text { the event }\end{array}$ \\
\hline$T$ & number of slots in a working or system cycle \\
\hline$D$ & number of slots that a sensor continues detecting the event \\
\hline$p$ & $\begin{array}{l}\text { probability that a sensor successfully detects the event in } \\
\text { an active slot }\end{array}$ \\
\hline$M(x, y)$ & $\begin{array}{l}\text { number of sensors that can detect the event when the } \\
\text { event occurs at location }(x, y)\end{array}$ \\
\hline$m_{i}$ & $\begin{array}{l}\text { number of sensors in } M(x, y) \text { that repeat their working } \\
\text { cycles at the } i \text { th slot in a system cycle }\end{array}$ \\
\hline$x_{i}$ & $\begin{array}{l}\text { number of sensors detect the event at the } i \text { th slot in a } \\
\text { working cycle }\end{array}$ \\
\hline$P_{k}\left(m_{1}, \cdots, m_{T}, a T+b\right)$ & $\begin{array}{l}\text { probability that there are at least } k \text { sensors succeeding in } \\
\text { detecting the event }\end{array}$ \\
\hline$N_{e}$ & $\begin{array}{l}\text { number of sensors that have ever succeeded in detecting } \\
\text { the event before the }(a T+b) \text { th slot }\end{array}$ \\
\hline$N_{f}$ & $\begin{array}{l}\text { number of sensors that first succeed in detecting the event } \\
\text { at the }(a T+b) \text { th slot }\end{array}$ \\
\hline$N_{1}$ & $\begin{array}{l}\text { number of sensors that have ever succeeded in detecting } \\
\text { the event before but do not detect at the }(a T+b) \text { th slot }\end{array}$ \\
\hline$N_{2}$ & $\begin{array}{l}\text { number of sensors that succeed in detecting the event at } \\
\text { the }(a T+b) \text { th slot }\end{array}$ \\
\hline$N_{3}$ & $\begin{array}{l}\text { number of sensors that have ever succeeded in detecting } \\
\text { the event before but fail at the }(a T+b) \text { th slot }\end{array}$ \\
\hline$S_{i}$ & number of sensors in the subset $i$ \\
\hline$R_{i}$ & $\begin{array}{l}\text { number of sensors that have succeeded in detecting the } \\
\text { event in the subset } i\end{array}$ \\
\hline
\end{tabular}


Theorem 1 The expected delay $\delta\left(m_{1}, \cdots, m_{T}\right)$ under the any-sensor-detection model is bounded by

$$
\delta\left(m_{1}, \cdots, m_{T}\right) \leq \frac{T^{2}}{(1-\alpha)^{2}}
$$

where $\alpha=(1-p)^{D \times M(x, y)}$.

PROOF. Since $\left(1-(1-p)^{x_{b}}\right) \leq 1$ and $(1-p) \leq 1$, we can obtain that

$$
\begin{aligned}
\delta\left(m_{1}, \cdots, m_{T}\right) & =\sum_{a=0}^{\infty} \sum_{b=1}^{T}(a T+b) \cdot\left(1-(1-p)^{x_{b}}\right)(1-p)^{a \times\left(x_{1}+\cdots+x_{T}\right)+x_{1}+\cdots+x_{b-1}} \\
& \leq \sum_{a=0}^{\infty} \sum_{b=1}^{T}(a T+b)(1-p)^{a \times\left(x_{1}+\cdots+x_{T}\right)} \\
& \leq \sum_{a=0}^{\infty}\left((1-p)^{a \times D \times M(x, y)} \sum_{b=1}^{T}(a+1) \times T\right) \\
& =\sum_{a=0}^{\infty} \alpha^{a}(a+1) T^{2} \\
& =\frac{T^{2}}{(1-\alpha)^{2}} .
\end{aligned}
$$

\section{2 $k$-Sensor-Detection Model}

Under this model, the event is considered to be captured by the network, once there are at least $k$ sensors successfully detecting its occurrence. Since the sequence $x_{1}, x_{2}, \ldots$ has a period of $T$, the expected latency can be written as

$$
\delta\left(m_{1}, \cdots, m_{T}\right)=\sum_{a=0}^{\infty} \sum_{b=1}^{T}(a T+b) \cdot P_{k}\left(m_{1}, \cdots, m_{T}, a T+b\right),
$$

where $P_{k}\left(m_{1}, \cdots, m_{T}, a T+b\right)$ is the probability that there are at least $k$ sensors successfully detecting the event at the $(a T+b)$ th slot, but not so before that slot. To find $P_{k}\left(m_{1}, \cdots, m_{T}, a T+b\right)$, let $N_{e}$ be the number of sensors that have already succeeded in detecting the event before the $(a T+b)$ th slot, and $N_{f}$ be the number of sensors that succeed in detecting this event at the $(a T+b)$ th slot for the first time. We first categorize sensors according to their behaviors as shown in Fig. 2. There are $x_{a T+b}=x_{b}$ active sensors at the $(a T+b)$ th slot, and the rest of $M(x, y)-x_{b}$ sensors are inactive. The inactive sensors can be further divided into a set of $N_{1}$ sensors which have ever succeeded in detecting this event before the $(a T+b)$ th slot, and a set of $M(x, y)-x_{b}-N_{1}$ sensors which have not. Similarly, the active sensors can be divided into a set 


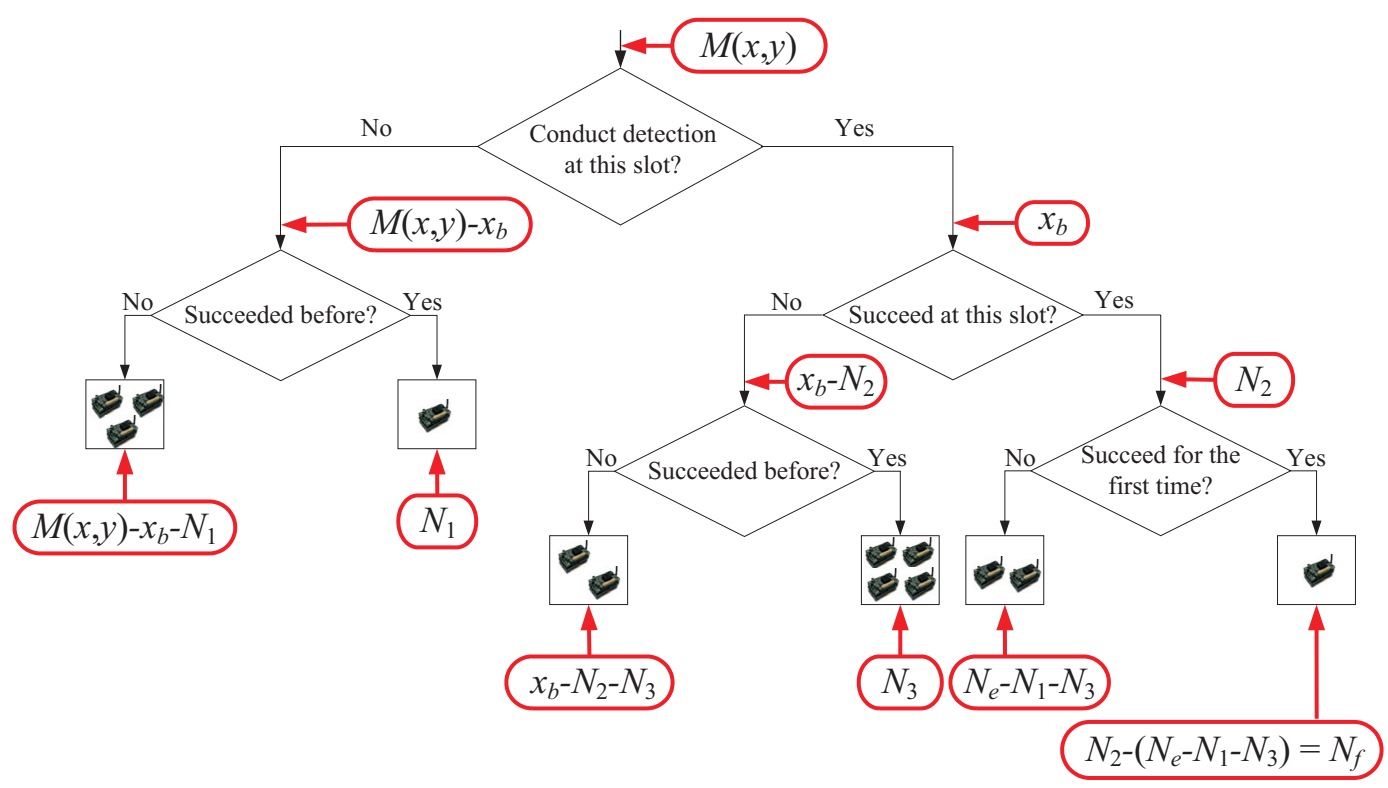

Fig. 2. Classification of sensors in the $(a T+b)$ th slot. Numbers in ovals indicate numbers of sensors.

of $N_{2}$ sensors which succeed in detecting this event at this slot, and a set of $x_{b}-N_{2}$ sensors which fail to detect this event at this slot. From the latter set, we further identify a set of $N_{3}$ sensors which have ever succeeded in detecting this event before the $(a T+b)$ th slot, but fail to detect this event at the current slot.

Based on the above definitions, once the values of $N_{e}, N_{1}, N_{2}$, and $N_{3}$ are given, the rest of the variables in Fig. 2 will all be fixed. Specifically, the number of sensors that successfully detect this event at the $(a T+b)$ th slot and have also succeeded in doing that before is $N_{e}-N_{1}-N_{3}$, and the number of sensors that succeed in detecting this event for the first time at the $(a T+b)$ th slot is $N_{f}=N_{2}-\left(N_{e}-N_{1}-N_{3}\right)$. In Eq. (3), the latency is considered to be $a T+b$ if $N_{e}<k$ and $N_{f}=\left(N_{1}+N_{2}+N_{3}\right)-N_{e} \geq k-N_{e}$. By enumerating all combinations of $N_{e}, N_{1}, N_{2}$, and $N_{3}$, we can derive that

$$
\begin{aligned}
P_{k}\left(m_{1}, \cdots, m_{T}, a T+b\right)= & \sum_{N_{e}=0}^{k-1}\left(\sum _ { h _ { 1 } = 0 } ^ { N _ { e } } \operatorname { P r o b } [ N _ { 1 } = h _ { 1 } ] \cdot \left(\sum_{h_{2}=0}^{x_{b}} \operatorname{Prob}\left[N_{2}=h_{2}\right] .\right.\right. \\
& \left.\left.\left(\sum_{h_{3}=0}^{N_{e}-h_{1}} \operatorname{Prob}\left[N_{3}=h_{3}\right] \cdot \operatorname{Prob}\left[N_{f} \geq k-N_{e}\right]\right)\right)\right) .
\end{aligned}
$$

Depending on the value of $b$, we can further derive the four terms $\operatorname{Prob}\left[N_{1}=h_{1}\right]$, $\operatorname{Prob}\left[N_{2}=h_{2}\right], \operatorname{Prob}\left[N_{3}=h_{3}\right]$, and $\operatorname{Prob}\left[N_{f} \geq k-N_{e}\right]$ with three cases.

Case (1): $b<D$. Consider the set of $M(x, y)-x_{b}$ inactive sensors at the $(a T+b)$ th slot. We divide them into two subsets: 
- $S_{1}$ : The set of sensors whose active phases do not cross the boundaries of system cycles.

- $S_{2}$ : The set of sensors whose active phases cross the boundaries of system cycles.

Clearly, $\left|S_{1}\right|=m_{b+1}+m_{b+2}+\ldots+m_{T-(D-1)}$ and $\left|S_{2}\right|=m_{T-(D-1)+1}+$ $m_{T-(D-1)+2}+\ldots+m_{T-(D-b)}$. For example, when $b=2$, Fig. 3 shows the above two subsets in case 1 . Recall the definition of $N_{1}$. Among these $N_{1}$ sensors, let $R_{1}$ be the number of sensors belonging to $S_{1}$, and $R_{2}$ the number of sensors belonging to $S_{2}$. Since $R_{1}+R_{2}=N_{1}$, we can expand Eq. (4) as follows:

$$
\begin{gathered}
P_{k}\left(m_{1}, \cdots, m_{T}, a T+b\right)=\sum_{N_{e}=0}^{k-1}\left(\sum_{h_{1}=0}^{N_{e}}\left(\sum_{r_{1}=0}^{h_{1}} \operatorname{Prob}\left[R_{1}=r_{1}\right] \cdot \operatorname{Prob}\left[R_{2}=h_{1}-r_{1}\right]\right) .\right. \\
\left.\left(\sum_{h_{2}=0}^{x_{b}} \operatorname{Prob}\left[N_{2}=h_{2}\right] \cdot\left(\sum_{h_{3}=0}^{N_{e}-h_{1}} \operatorname{Prob}\left[N_{3}=h_{3}\right] \cdot \operatorname{Prob}\left[N_{f} \geq k-N_{e}\right]\right)\right)\right) .
\end{gathered}
$$

Given two integers $x$ and $y$ such that $x \geq y$ and a probability value $z$, let us define

$$
\operatorname{Bino}(x, y, z)=\left(\begin{array}{l}
x \\
y
\end{array}\right) z^{y} \cdot(1-z)^{x-y} .
$$

The probability functions in Eq. (5) are derived as follows:

$$
\begin{aligned}
& \operatorname{Prob}\left[R_{1}=r_{1}\right]=\operatorname{Bino}\left(\left|S_{1}\right|, r_{1}, 1-(1-p)^{a D}\right), \\
& \operatorname{Prob}\left[R_{2}=h_{1}-r_{1}\right]=\sum_{i=1}^{D-2} \frac{m_{T-(D-1)+i}}{\left|S_{2}\right|} \cdot \operatorname{Bino}\left(\left|S_{2}\right|, h_{1}-r_{1}, 1-(1-p)^{a D+i}\right), \\
& \operatorname{Prob}\left[N_{2}=h_{2}\right]=\operatorname{Bino}\left(x_{b}, h_{2}, p\right), \\
& \operatorname{Prob}\left[N_{3}=h_{3}\right]=\operatorname{Bino}\left(x_{b}-h_{2}, h_{3}, \sum_{i=0}^{b-1} \frac{m_{b-i}}{x_{b}}\left(1-(1-p)^{a D+b-i}\right)+\right. \\
& \left.\quad \sum_{i=0}^{D-b-1} \frac{m_{T-i}}{x_{b}}\left(1-(1-p)^{a D+b}\right)\right), \text { and } \\
& \operatorname{Prob}\left[N_{f} \geq k-N_{e}\right]=\operatorname{Bino}\left(h_{2}, N_{f}, \sum_{i=0}^{b-1} \frac{m_{b-i}}{x_{b}}(1-p)^{a D+b-i}+\right. \\
& \left.\sum_{i=0}^{D-b-1} \frac{m_{T-i}}{x_{b}}(1-p)^{a D+b-1}\right) .
\end{aligned}
$$

$\operatorname{Prob}\left[R_{1}=r_{1}\right]$ is the probability that $r_{1}$ sensors in $S_{1}$ have ever succeeded in detecting this event before the $(a T+b)$ th slot, where $1-(1-p)^{a D}$ is the probability that such a sensor has ever successfully detected this event before the $(a T+b)$ th slot. $\operatorname{Prob}\left[R_{2}=h_{1}-r_{1}\right]$ is derived similarly, except that we are concerned about sensors in $S_{2}$ and, among these sensors, there is a ratio of $\frac{m_{T-(D-1)+i}}{\left|S_{2}\right|}$ of sensors which have tried to detect this event for $a D+i$ slots 


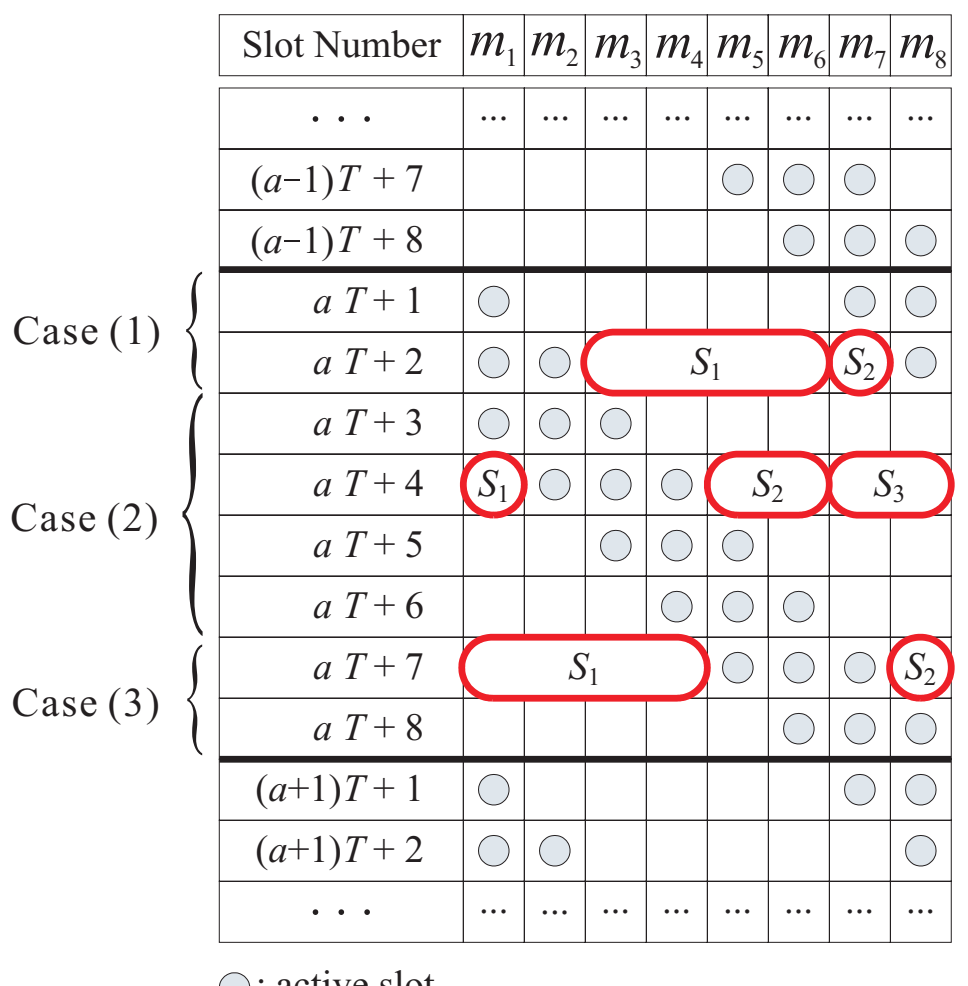

Fig. 3. Classification of sensors in a network with $T=8$ and $D=3$.

(and we take their average). $\operatorname{Prob}\left[N_{2}=h_{2}\right]$ is the probability that there are $h_{2}$ sensors among $x_{b}$ sensors successfully detecting the event at the $(a T+b)$ th slot. $\operatorname{Prob}\left[N_{3}=h_{3}\right]$ is the probability that there are $h_{3}$ sensors among $x_{b}-h_{2}$ sensors that have ever successfully detected the event before the $(a T+b)$ th slot. Note that the third term in $\operatorname{Bino}(\cdot)$ is to take care of those sensors whose active slots do not (the first expression) and do (the second expression) cross the boundaries of system cycles, and we take their average. $\operatorname{Prob}\left[N_{f} \geq k-N_{e}\right]$ is similar to the previous probability except that these sensors succeed for the first time at the $(a T+b)$ th slot.

Case (2): $D \leq b \leq T-D+1$. In this case, we divide the set of inactive $M(x, y)-x_{b}$ sensors at the $(a T+b)$ th slot into three subsets according to whether their active slots cross the boundaries of system cycles:

- $S_{1}$ : The set of sensors which have finished their active slots in the current system cycle and whose active slots do not cross the boundaries of system cycles.

- $S_{2}$ : The set of sensors which have not started their active slots in the current system cycle and whose active slots do not cross the boundaries of system cycles.

- $S_{3}$ : The set of sensors whose active slots cross the boundaries of system cycles. 
We can obtain that $\left|S_{1}\right|=\sum_{i=1}^{b-D} m_{i},\left|S_{2}\right|=\sum_{i=b+1}^{T-(D-1)} m_{i}$, and $\left|S_{3}\right|=\sum_{i=T-(D-1)+1}^{T} m_{i}$. For example, when $b=4$, Fig. 3 shows these subsets in case 2. Again, let $R_{3}$ be the number of sensors belonging to $S_{3}$. Since $R_{1}+R_{2}+R_{3}=S_{1}$, we can expand Eq. (4) as follows:

$$
\begin{gathered}
P_{k}\left(m_{1}, \cdots, m_{T}, a T+b\right)=\sum_{N_{e}=0}^{k-1}\left(\sum _ { h _ { 1 } = 0 } ^ { N _ { e } } \left(\sum_{r_{1}=0}^{h_{1}} \sum_{r_{2}=0}^{h_{1}-r_{1}} \operatorname{Prob}\left[R_{1}=r_{1}\right] \cdot\right.\right. \\
\left.\operatorname{Prob}\left[R_{2}=r_{2}\right] \cdot \operatorname{Prob}\left[R_{3}=h_{1}-r_{1}-r_{2}\right]\right) \cdot\left(\sum_{h_{2}=0}^{x_{b}} \operatorname{Prob}\left[N_{2}=h_{2}\right] \cdot\right. \\
\left.\left.\left(\sum_{h_{3}=0}^{N_{e}-h_{1}} \operatorname{Prob}\left[N_{3}=h_{3}\right] \cdot \operatorname{Prob}\left[N_{f} \geq k-N_{e}\right]\right)\right)\right),
\end{gathered}
$$

where

$$
\begin{aligned}
& \operatorname{Prob}\left[R_{1}=r_{1}\right]=\operatorname{Bino}\left(\left|S_{1}\right|, r_{1}, 1-(1-p)^{(a+1) D}\right), \\
& \operatorname{Prob}\left[R_{2}=r_{2}\right]=\operatorname{Bino}\left(\left|S_{2}\right|, r_{2}, 1-(1-p)^{a D}\right), \\
& \operatorname{Prob}\left[R_{3}=h_{1}-r_{1}-r_{2}\right]=\sum_{i=0}^{D-2} \frac{m_{T-(D-1)+1+i}}{\left|S_{3}\right|} . \\
& \operatorname{Bino}\left(\left|S_{3}\right|, h_{1}-r_{1}-r_{2}, 1-(1-p)^{a D+i+1}\right), \\
& \operatorname{Prob}\left[N_{2}=h_{2}\right]=\operatorname{Bino}\left(x_{b}, h_{2}, p\right), \\
& \operatorname{Prob}\left[N_{3}=h_{3}\right]=\operatorname{Bino}\left(x_{b}-h_{2}, h_{3}, \sum_{i=0}^{D-1} \frac{m_{b-i}}{x_{b}}\left(1-(1-p)^{a D+i}\right)\right), \text { and } \\
& \operatorname{Prob}\left[N_{f} \geq k-N_{e}\right]=\operatorname{Bino}\left(h_{2}, N_{f}, \sum_{i=0}^{D-1} \frac{m_{b-i}}{x_{b}}(1-p)^{a D+i}\right) .
\end{aligned}
$$

Again, $\operatorname{Prob}\left[R_{3}=h_{1}-r_{1}-r_{2}\right]$ is the probability that $h_{1}-r_{1}-r_{2}$ sensors in $S_{3}$ have ever succeeded in detecting this event before the $(a T+b)$ th slot, where $1-(1-p)^{a D+i+1}$ is the probability that such a sensor have ever successfully detected this event before the $(a T+b)$ th slot. However, among these sensors in $S_{3}$, there is a ratio of $\frac{m_{T-(D-1)+1+i}}{\left|S_{3}\right|}$ of sensors which have tried to detect this event for $a D+i$ slots, and thus we take their average.

Case (3): $b>T-D+1$. In this case, we divide the set of inactive $M(x, y)-x_{b}$ sensors at the $(a T+b)$ th slot into two subsets according to whether their active slots cross the boundaries of system cycles:

- $S_{1}$ : The set of sensors whose active slots do not cross the boundaries of system cycles.

- $S_{2}$ : The set of sensors whose active slots cross the boundaries of system cycles.

We have $S_{1}=\sum_{i=1}^{b-D} m_{i}$ and $S_{2}=\sum_{i=b+1}^{T} m_{i}$. Fig. 3 gives an example when 
$b=7$. We derive Eq. (4) as follows:

$$
\begin{array}{r}
P_{k}\left(m_{1}, \cdots, m_{T}, a T+b\right)=\sum_{N_{e}=0}^{k-1}\left(\sum_{h_{1}=0}^{N_{e}}\left(\sum_{r_{1}=0}^{h_{1}} \operatorname{Prob}\left[R_{1}=r_{1}\right] \cdot \operatorname{Prob}\left[R_{2}=h_{1}-r_{1}\right]\right) .\right. \\
\left.\left(\sum_{h_{2}=0}^{x_{b}} \operatorname{Prob}\left[N_{2}=h_{2}\right] \cdot\left(\sum_{h_{3}=0}^{N_{e}-h_{1}} \operatorname{Prob}\left[N_{3}=h_{3}\right] \cdot \operatorname{Prob}\left[N_{f} \geq k-N_{e}\right]\right)\right)\right),
\end{array}
$$

where

$$
\begin{aligned}
& \operatorname{Prob}\left[R_{1}=r_{1}\right]=\operatorname{Bino}\left(\left|S_{1}\right|, r_{1}, 1-(1-p)^{(a+1) D}\right), \\
& \operatorname{Prob}\left[R_{2}=h_{1}-r_{1}\right]=\sum_{i=1}^{D-2} \frac{m_{T-(D-1)+1+i}}{\left|S_{2}\right|} \cdot \operatorname{Bino}\left(\left|S_{2}\right|, h_{1}-r_{1}, 1-(1-p)^{a D+i+1}\right), \\
& \operatorname{Prob}\left[N_{2}=h_{2}\right]=\operatorname{Bino}\left(x_{b}, h_{2}, p\right), \\
& \operatorname{Prob}\left[N_{3}=h_{3}\right]=\operatorname{Bino}\left(x_{b}-h_{2}, h_{3}, \sum_{i=0}^{T-b} \frac{m_{T-(D-1)-i}}{x_{b}}\left(1-(1-p)^{a D+i+1}\right)+\right. \\
& \left.\quad \sum_{i=0}^{b-T+(D-1)-1} \frac{m_{T-(D-1)+1+i}}{x_{b}}\left(1-(1-p)^{a D+i+1}\right)\right), \text { and } \\
& \operatorname{Prob}\left[N_{f} \geq k-N_{e}\right]=\operatorname{Bino}\left(h_{2}, N_{f},\right. \\
& \left.\quad \sum_{i=0}^{T-b} \frac{m_{T-(D-1)-i}}{x_{b}}(1-p)^{a D+i+1}+\sum_{i=0}^{b-T+(D-1)-1} \frac{m_{T-(D-1)+1+i}}{x_{b}}(1-p)^{a D+i+1}\right) .
\end{aligned}
$$

Finally, by replacing $P_{k}\left(m_{1}, \cdots, m_{T}, a T+b\right)$ in Eq. (3) with one of the above three cases, we can obtain the expected latency $\delta\left(m_{1}, \cdots, m_{T}\right)$ under the $k$ sensor-detection model.

Table 2 summarizes the four terms $\operatorname{Prob}\left[N_{1}=h_{1}\right], \operatorname{Prob}\left[N_{2}=h_{2}\right], \operatorname{Prob}\left[N_{3}=h_{3}\right]$, and $\operatorname{Prob}\left[N_{f} \geq k-N_{e}\right]$ in Eq. (4) under the three cases.

\section{Using Detection Latency to Guide Deployment}

Event detection latency can be used as an index to evaluate a WSN's coverage and thus can help guide the deployment of a WSN. Below, we briefly discuss how to improve the coverage of a WSN. First, we can partition the sensing field into several subregions. Then, we can evaluate the event detection latency of each subregion. If the expected latency of a region is larger than a tolerable threshold, it means that there are not enough sensors deployed in the region. For such regions, we can deploy more sensors to improve their expected detection latencies.

Beside, the event detection latency can also be used to measure the latency 
Table 2

Summary of the four terms $\operatorname{Prob}\left[N_{1}=h_{1}\right], \operatorname{Prob}\left[N_{2}=h_{2}\right], \operatorname{Prob}\left[N_{3}=h_{3}\right]$, and $\operatorname{Prob}\left[N_{f} \geq k-N_{e}\right]$ in Eq. (4) under different cases, where $q=1-p$.

\begin{tabular}{|c|c|}
\hline Cases & Terms \\
\hline$b<D$ & $\begin{array}{l}\operatorname{Prob}\left[N_{1}=h_{1}\right]=\sum_{r_{1}=0}^{h_{1}} \operatorname{Bino}\left(\left|S_{1}\right|, r_{1}, 1-q^{a D}\right) . \\
\quad \sum_{i=1}^{D-2} \frac{m_{T-D+i+1}}{\left|S_{2}\right|} \operatorname{Bino}\left(\left|S_{2}\right|, h_{1}-r_{1}, 1-q^{a D+i}\right) \\
\operatorname{Prob}\left[N_{2}=h_{2}\right]=\operatorname{Bino}\left(x_{b}, h_{2}, p\right) \\
\operatorname{Prob}\left[N_{3}=h_{3}\right]=\operatorname{Bino}\left(x_{b}-h_{2}, h_{3}, \sum_{i=0}^{b-1} \frac{m_{b-i}}{x_{b}}\left(1-q^{a D+b-i}\right)\right. \\
\left.\quad+\sum_{i=0}^{D-b-1} \frac{m_{T-i}}{x_{b}}\left(1-q^{a D+b}\right)\right) \\
\operatorname{Prob}\left[N_{f} \geq k-N_{e}\right]=\operatorname{Bino}\left(h_{2}, N_{f}, \sum_{i=0}^{b-1} \frac{m_{b-i}}{x_{b}} q^{a D+b-i}+\right. \\
\left.\quad \sum_{i=0}^{D-b-1} \frac{m_{T-i}}{x_{b}} q^{a D+b-1}\right)\end{array}$ \\
\hline$D \leq b \leq T-D+1$ & $\begin{array}{l}\operatorname{Prob}\left[N_{1}=h_{1}\right]=\sum_{r_{1}=0}^{h_{1}} \sum_{r_{2}=0}^{h_{1}-r_{1}} \operatorname{Bino}\left(\left|S_{1}\right|, r_{1}, 1-q^{(a+1) D}\right) \cdot \\
\quad \operatorname{Bino}\left(\left|S_{2}\right|, r_{2}, 1-q^{a D}\right) \cdot \sum_{i=0}^{b-2} \frac{m_{T-D+i+2}}{\left|S_{3}\right|} . \\
\quad \operatorname{Bino}\left(\left|S_{3}\right|, h_{1}-r_{1}-r_{2}, 1-q^{a D+i+1}\right) \\
\operatorname{Prob}\left[N_{2}=h_{2}\right]=\operatorname{Bino}\left(x_{b}, h_{2}, p\right) \\
\operatorname{Prob}\left[N_{3}=h_{3}\right]=\operatorname{Bino}\left(x_{b}-h_{2}, h_{3}, \sum_{i=0}^{D-1} \frac{m_{b-i}}{x_{b}}\left(1-q^{a D+i}\right)\right) \\
\operatorname{Prob}\left[N_{f} \geq k-N_{e}\right]=\operatorname{Bino}\left(h_{2}, N_{f}, \sum_{i=0}^{D-1} \frac{m_{b-i}}{x_{b}} q^{a D+i}\right)\end{array}$ \\
\hline$b>T-D+1$ & $\begin{array}{l}\operatorname{Prob}\left[N_{1}=h_{1}\right]=\sum_{r_{1}=0}^{h_{1}} \operatorname{Bino}\left(\left|S_{1}\right|, r_{1}, 1-q^{(a+1) D}\right) \\
\quad \sum_{i=1}^{D-2} \frac{m_{T-D+i+2}}{\left|S_{2}\right|} \operatorname{Bino}\left(\left|S_{2}\right|, h_{1}-r_{1}, 1-q^{a D+i+1}\right) \\
\operatorname{Prob}\left[N_{2}=h_{2}\right]=\operatorname{Bino}\left(x_{b}, h_{2}, p\right) \\
\operatorname{Prob}\left[N_{3}=h_{3}\right]=\operatorname{Bino}\left(x_{b}-h_{2}, h_{3}, \sum_{i=0}^{T-b} \frac{T-D-i+1}{x_{b}} .\right. \\
\left.\quad\left(1-q^{a D+i+1}\right)+\sum_{i=0}^{b+D-T-2} \frac{m_{T-D+i+2}}{x_{b}}\left(1-q^{a D+i+1}\right)\right) \\
\operatorname{Prob}\left[N_{f} \geq k-N_{e}\right]=\operatorname{Bino}\left(h_{2}, N_{f}, \sum_{i=0}^{T-b} \frac{T-D-i+1}{x_{b}} q^{a D+i+1}\right. \\
\left.\quad+\sum_{i=0}^{b+D-T-2} \frac{m_{T-D+i+2}}{x_{b}} q^{a D+i+1}\right)\end{array}$ \\
\hline
\end{tabular}

to detect a node newly joining a wireless personal area network (WPAN). We observe that for a device to join a WPAN, usually a network discovery procedure needs to be taken. To facilitate network discovery, coordinators in a WPAN normally need to send beacons periodically to announce their presence (for example, Bluetooth, WiMedia [22], and ZigBee follow this model). If we regard the beacon windows as our active phases, then the event detection latency under our any-sensor-detection model is the latency for a new node to discover the WPAN. 


\section{Simulation Results}

We have developed a simulator using $\mathrm{C}++$ language to verify our analytical results. In the simulations, we set up a sensing field of size $10 \times 10$, on which there are 50 sensors randomly deployed. Each sensor has a sensing distance of 3 units. Events may appear in any location inside the sensing field. Given a network configuration, we evaluate the event detection latency by both Eq. (1) and the simulations. For each simulation, at least 1000 experiments are repeated, and we take their average.

Fig. 4 shows the event detection latencies under different values of detection probability $p$. The simulation results coincide well with the analytical results, except when $p=0.1$ under the 5 -sensor-detection model. This is because the simulator only simulates 1000 possible locations that an event may occur, while the analysis (Eq. (1)) has to consider all possible locations inside the sensing field. Since the value of $p$ is small, the network requires longer time to capture the event than we expect.

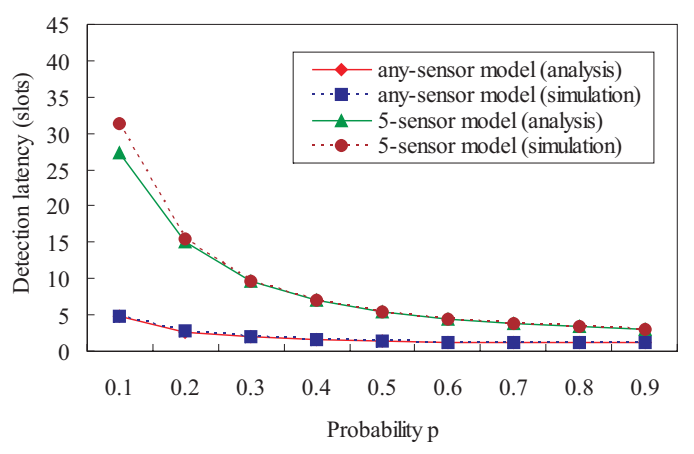

(a) $\mathrm{T}=5$ and $\mathrm{D}=1$

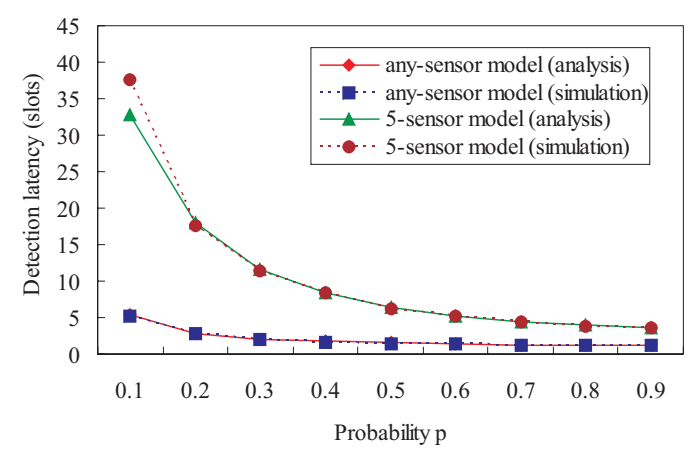

(c) $\mathrm{T}=5$ and $\mathrm{D}=1$

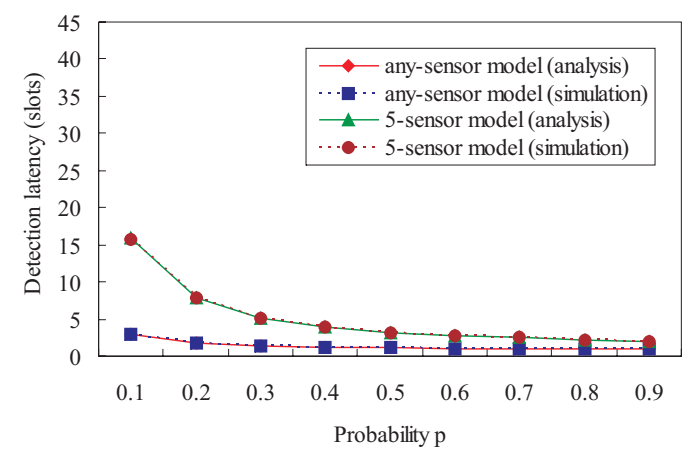

(b) $\mathrm{T}=5$ and $\mathrm{D}=2$

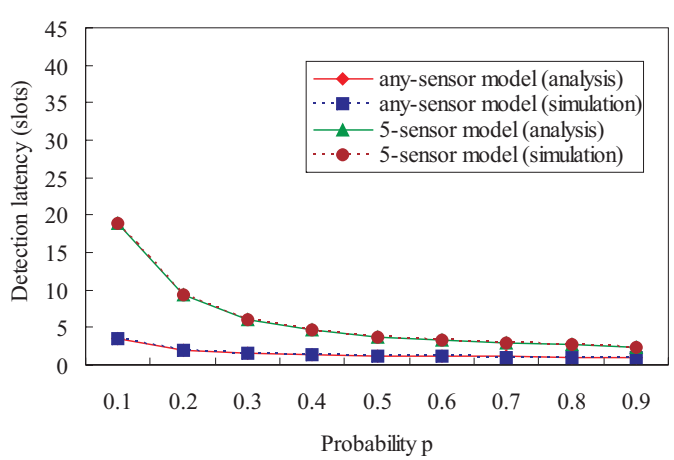

(d) $\mathrm{T}=5$ and $\mathrm{D}=2$

Fig. 4. The event detection latencies under different values of probability $p$.

Fig. 5 shows the event detection latencies under different values of $M(x, y)$. In the simulation, when an event occurs within $i$ sensors' sensing ranges, we record the detection latency in the corresponding $M(x, y)=i$ statistics. From Fig. 5, we can observe that the simulation results coincide well with the analytical results, except when $p=0.1$ and $M(x, y) \leq 5$ under 3 -sensor-detection 
model. This is because our analysis assumes a larger-scale network. It can be observed that a larger $M(x, y)$, which implies a higher network density, can help reduce the detection latency. A larger detection probability $p$, which reflects the sensibility of sensors, can also reduce the detection latency. The result can be used to determine how sensors should be arranged at the deployment stage.

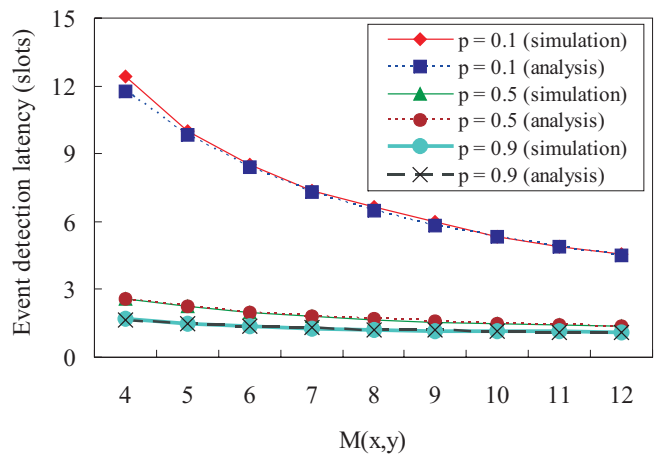

(a) $\mathrm{T}=5$ and $\mathrm{D}=1$ (any-sensor model)

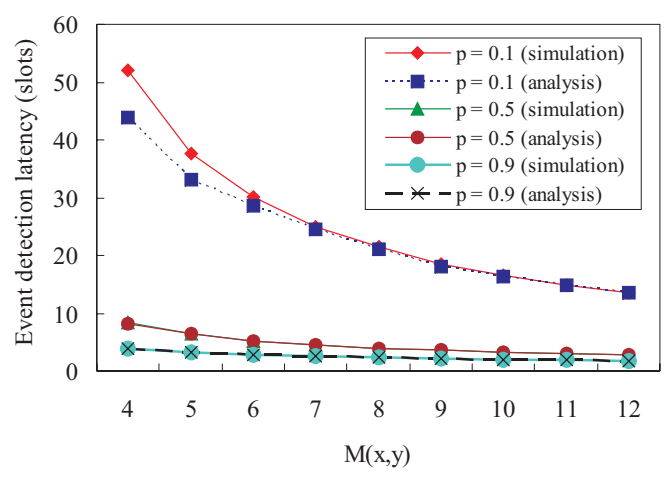

(c) $\mathrm{T}=5$ and $\mathrm{D}=1(3$-sensor model $)$

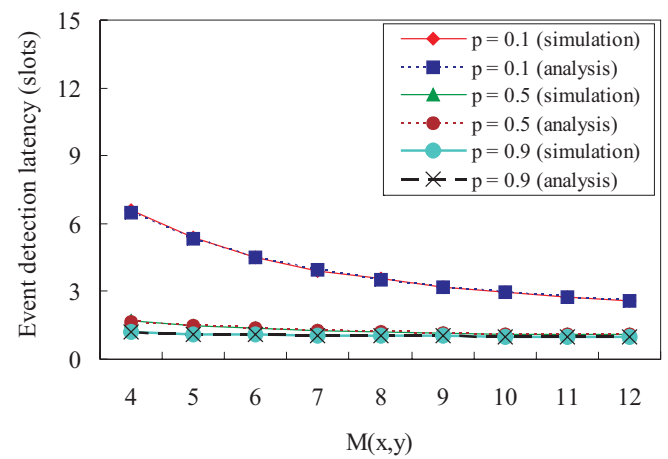

(b) $\mathrm{T}=5$ and $\mathrm{D}=2$ (any-sensor model)

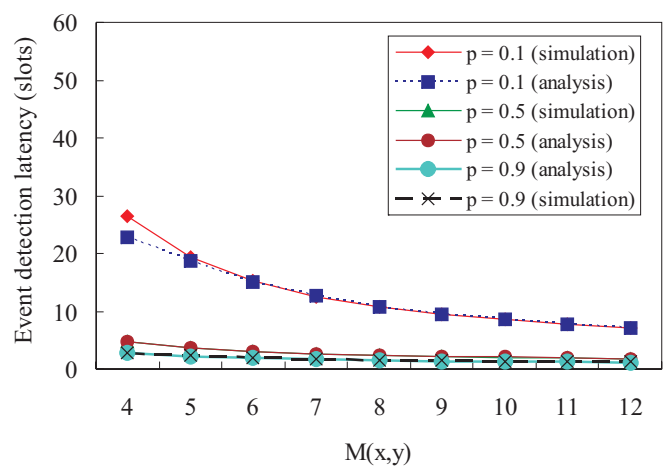

(d) $\mathrm{T}=5$ and $\mathrm{D}=2(3$-sensor model $)$

Fig. 5. The event detection latencies under different values of $M(x, y)$.

In both Figs. 4 and 5, we can observe that the event detection latency can be greatly reduced when we increase the number of active slots $D$, especially when the detection probability $p$ is small. Thus, we have interest in observing the effect of $D$ on the event detection latency under different values of $M(x, y)$ and $p$, as shown in Fig. 6. To show the effect of $D$, we set the period $T$ as a constant of 16 slots. From Fig. 6, we can observe that the latencies drop as the value of $D$ increases, but this effect becomes less significant when $D \geq 4$. Since a sensor will consume more energy as the length of active slots $D$ increases, this result can be used to decide the length of a sensor's active phase to reduce both event detection latency and energy consumption of a WSN. 


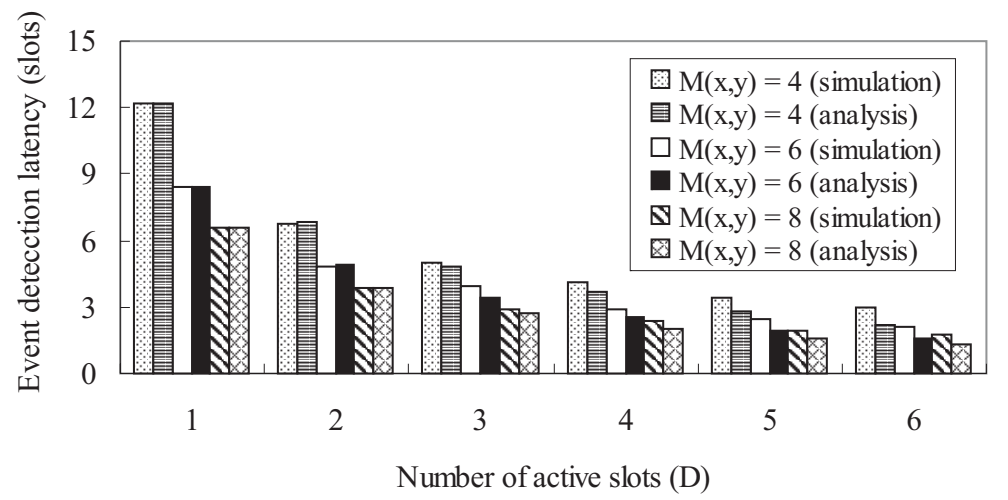

(a) $p=0.3$ (any-sensor model)

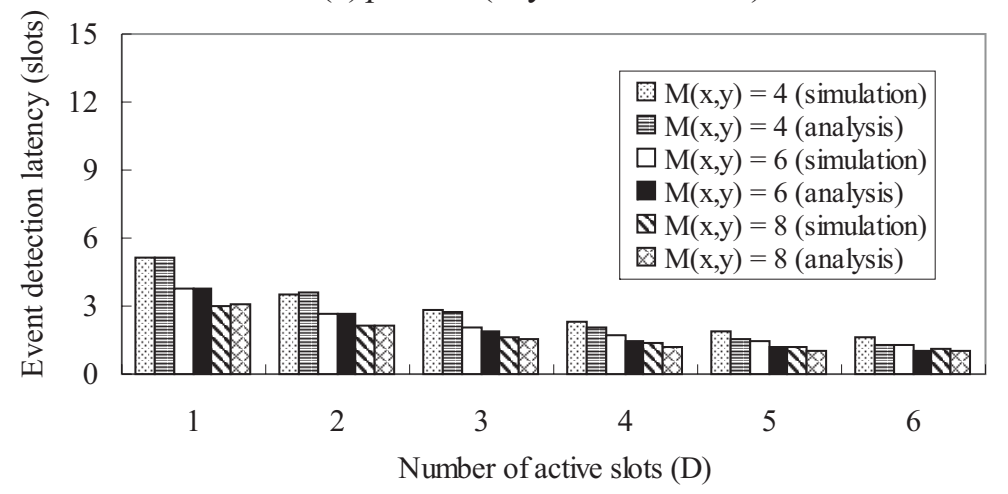

(b) $p=0.7$ (any-sensor model)

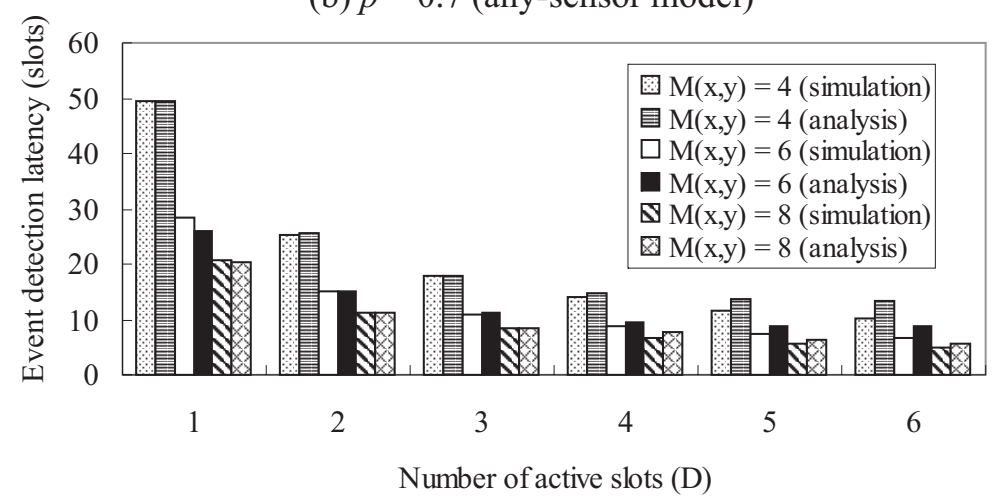

(c) $p=0.3$ (3-sensor model)

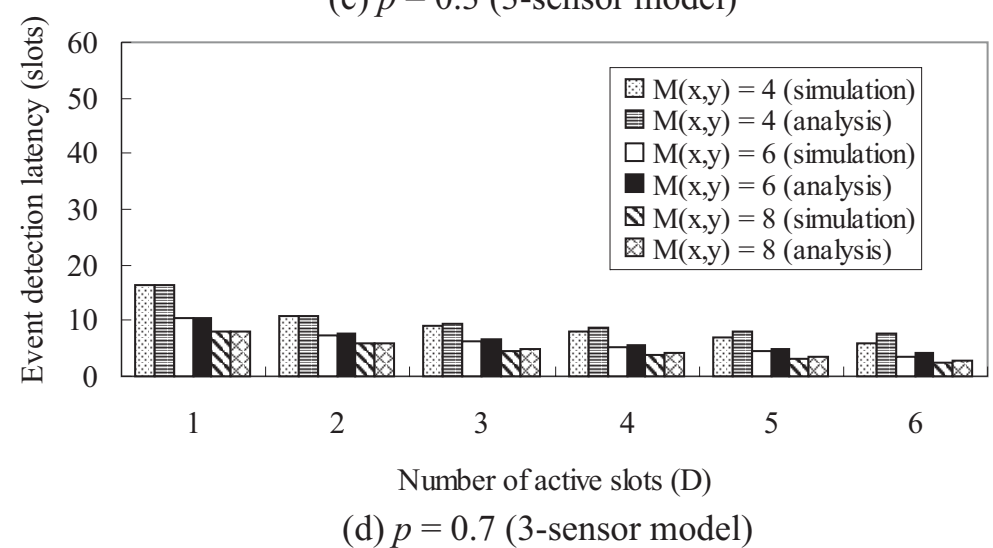

Fig. 6. The event detection latencies under different values of $D(T=16)$. 


\section{Conclusions}

We have proposed a methodology to analyze the event detection latency of a WSN. Such a latency analysis can be used to measure the network coverage and the time that a new node needs to discover a network. We have adopted a probabilistic approach to analyze the latency under an any-sensor-detection and a $k$-sensor-detection models. We have also developed a simulator to verify our analyses. Simulation results not only coincide well with the analyses, but also indicate the potential factors that affect the latency.

Our analysis assumes that the detection probability $p$ is a constant. It deserves to further study the same problem when the value of $p$ is a function of the distance between a sensor and the event. Also, our analysis models time in a discrete manner (by fixed-length slots). It is also interesting to investigate the continuous time case.

\section{Acknowledgements}

Y. C. Tseng's research is co-sponsored by Taiwan's MOE ATU Program, by NSC under grant numbers 93-2752-E-007-001-PAE, 95-2623-7-009-010-ET, 952218-E-009-020, 95-2219-E-009-007, 94-2213-E-009-004, and 94-2219-E-007009, by MOEA under grant number 94-EC-17-A-04-S1-044, by ITRI, Taiwan, and by Intel Inc.

\section{References}

[1] I. F. Akyildiz, W. Su, Y. Sankarasubramaniam, E. Cayirci, A survey on sensor networks, IEEE Communications Magazine 40 (8) (2002) 102-114.

[2] D. Niculescu, Communication paradigms for sensor networks, IEEE Communications Magazine 43 (3) (2005) 116-122.

[3] J. Burrell, T. Brooke, R. Beckwith, Vineyard computing: sensor networks in agricultural production, IEEE Pervasive Computing 3 (1) (2004) 38-45.

[4] K. Chintalapudi, T. Fu, J. Paek, N. Kothari, S. Rangwala, J. Caffrey, R. Govindan, E. Johnson, S. Masri, Monitoring civil structures with a wireless sensor network, IEEE Internet Computing 10 (2) (2006) 26-34.

[5] T. T. Hsieh, Using sensor networks for highway and traffic applications, IEEE Potentials 23 (2) (2004) 13-16. 
[6] A. Mainwaring, D. Culler, J. Polastre, R. Szewczyk, J. Anderson, Wireless sensor networks for habitat monitoring, in: ACM International Workshop on Wireless Sensor Networks and Applications, 2002, pp. 88-97.

[7] R. Szewczyk, E. Osterweil, J. Polastre, M. Hamilton, A. Mainwaring, D. Estrin, Habitat monitoring with sensor networks, Communications of the ACM 47 (6) (2004) 34-40.

[8] P. K. Biswas, S. Phoha, Self-organizing sensor networks for integrated target surveillance, IEEE Transactions on Computers 55 (8) (2006) 1033-1047.

[9] T. He, S. Krishnamurthy, L. Luo, T. Yan, L. Gu, R. Stoleru, G. Zhou, Q. Cao, P. Vicaire, J. A. Stankovic, T. F. Abdelzaher, J. Hui, B. Krogh, VigilNet: An integrated sensor network system for energy-efficient surveillance, ACM Transactions on Sensor Networks 2 (1) (2006) 1-38.

[10] Y. C. Tseng, Y. C. Wang, K. Y. Cheng, An integrated mobile surveillance and wireless sensor (iMouse) system and its detection delay analysis, in: ACM International Symposium on Modeling, Analysis and Simulation of Wireless and Mobile Systems, 2005, pp. 178-181.

[11] C. Gui, P. Mohapatra, Power conservation and quality of surveillance in target tracking sensor networks, in: ACM International Conference on Mobile Computing and Networking, 2004, pp. 129-143.

[12] H. T. Kung, D. Vlah, Efficient location tracking using sensor networks, in: Wireless Communications and Networking, 2003, pp. 1954-1961.

[13] W. Zhang, G. Cao, DCTC: dynamic convoy tree-based collaboration for target tracking in sensor networks, IEEE Transactions on Wireless Communications 3 (5) (2004) 1689-1701.

[14] LAN/MAN Standards Committee of the IEEE Computer Society, IEEE Std 802.15.4-2003, Wireless medium access control (MAC) and physical layer (PHY) specifications for low-rate wireless personal area networks (LR-WPANs), IEEE.

[15] LAN/MAN Standards Committee of the IEEE Computer Society, IEEE Std 802.15.1-2005, Wireless medium access control (MAC) and physical layer (PHY) specifications for wireless personal area networks (WPANs), IEEE.

[16] W. Ye, J. Heidemann, D. Estrin, Medium access control with coordinated adaptive sleeping for wireless sensor networks, IEEE/ACM Transactions on Networking 12 (3) (2004) 493-506.

[17] S. S. Dhillon, K. Chakrabarty, Sensor placement for effective coverage and surveillance in distributed sensor networks, in: IEEE Wireless Communications and Networking, 2003, pp. 1609-1614.

[18] Y. Zou, K. Chakrabarty, A distributed coverage- and connectivity-centric technique for selecting active nodes in wireless sensor networks, IEEE Transactions on Computers 54 (2005) 978-991. 
[19] A. Nasipuri, K. Li, A directionality based location discovery scheme for wireless sensor networks, in: ACM International Workshop on Wireless Sensor Networks and Applications, 2002, pp. 105-111.

[20] D. Nicules, B. Nath, Ad-hoc positioning system (APS) using AoA, in: IEEE INFOCOM, 2003, pp. 1734-1743.

[21] A. Savvides, C. C. Han, M. B. Strivastava, Dynamic fine-grained localization in ad-hoc networks of sensors, in: International Conference on Mobile Computing and Networking, 2001, pp. 166-179.

[22] LAN/MAN Standards Committee of the IEEE Computer Society, IEEE Std 802.15.3-2003, Wireless medium access control (MAC) and physical layer (PHY) specifications for high rate wireless personal area networks (WPANs), IEEE. 